\title{
UMA REPRESENTAÇÃO S-DIGRAPH PARA AS FUNÇÕES CARACTERÍSTICAS DE MASSIEU
}

\author{
UNA REPRESENTACIÓN S-DIGRAPH PARA EL FUNCIONES \\ CARACTERÍSTICAS DE MASSIEU
}

\begin{abstract}
A S-DIGRAPH REPRESENTATION FOR THE FUNCTIONS CHARACTERISTICS OF MASSIEU
\end{abstract}

DOI: $10.22481 /$ rbba.v10i02.9684

Jornandes Jesús Correia Universidade Estadual do Sudoeste da Bahia, Bahia, Brasil Lattes: http://lattes.cnpq.br/5594644574555987

ORCID: https://orcid.org/0000-0002-0035-2300 Endereço eletrônico: jornandes.correia@uesb.edu.br

\section{RESUMO}

Este artigo propõe um modelo cúbico graficodirecionado de Sistemas Simples na representação da Entropia para as Funções Características de Massieu, articuladas por Equações de Estado, tendo como referência o Cubo de Correia na representação da Energia e baseado no Octaedro de Koenig, no Cuboctaedro de Fox, no Cubo Termodinâmico de Pate, do Esquema Mnemônico de Zhao, do Diagrama Termodinâmico de Venn, no Octaedro de Kerr \& Makosko, nos Grafos de Pogliani e no Cubo Mágico de Kocik. A energia, por unidade de temperatura, da Equação de estado é proveniente do produto entre duas variáveis conjugadas pela Seta Coordenada que estiverem na mesma direção da Seta Aresta que vincula as duas Funções Características. As equações fundamentais dos potenciais termodinâmicos revelam que a Entropia de um sistema termodinâmico é a razão entre a sua Energia 
Térmica pela Temperatura Absoluta. Pretende-se, com este modelo, facilitar a elaboração dos construtos lógicos e a compreensão da interdependência entre os Potenciais da Termodinâmica e estabecer um sentido para as Transformações Termodinâmicas.

Palavras-chave: Funções Características. Cubo Termodinâmico. Ensino da Termodinâmica. Representação S-Digraph.

\title{
RESUMEN
}

Este artículo propone un modelo cúbico dirigido por grafos de Sistemas Simples en la representación de Entropía para Funciones Características de Massieu articulado por Ecuaciones de Estado, teniendo como referencia el Cubo de Correia en la representación de Energía y basado en el Octaedro de Koenig, el Cuboctaedro de Fox, el Cubo Termodinámico de Pate, el Esquema Nemónico de Zhao, el Diagrama Termodinámico de Venn y el Octaedro Kerr \& Makosko de Venn, en Pogliani Graphics y en Kocik's Magic Cube. La energía, por unidad de temperatura, de la Ecuación de Estado proviene del producto entre dos variables conjugadas por la Flecha de Coordenadas que está en la misma dirección que la Flecha de Borde que conecta las dos Funciones Características. Las ecuaciones fundamentales de los potenciales termodinámicos revelan que la entropía de un sistema termodinámico es la relación entre su energía térmica y la temperatura absoluta. El objetivo de este modelo es facilitar la elaboración de constructos lógicos y la comprensión de la interdependencia entre los Potenciales de la Termodinámica y establecer un significado para las Transformaciones Termodinámicas.

Palabras clave: Funciones Características. Cubo Termodinámico; Enseñanza de la Termodinámica. Representación S-Digraph.

\begin{abstract}
This article proposes a graphical-directed cubic model of Simple Systems in the representation of Entropy for Massieu's Characteristic Functions, articulated by Equations of State, having as reference the Cube of Correia in the representation of Energy and based on Koenig's Octahedron, in Fox's Cuboctahedron, in Pate's Thermodynamic Cube, Zhao's Mnemonic Schema, Venn's Thermodynamic Diagram, Kerr \& Makosko's Octahedron, Pogliani's Graphs, and Kocik's Magic Cube. The energy,
\end{abstract}


divided by temperature, of the Equation of State comes from the product between two variables conjugated by the Coordinate Arrow that is in the same direction as the Edge Arrow that links the two Characteristic Functions. The fundamental equations of thermodynamic potentials reveal that the Entropy of a thermodynamic system is the ratio of its Thermal Energy by the Absolute Temperature. The aim of this model is to facilitate the elaboration of logical constructs and the understanding of the interdependence between the Potentials of Thermodynamics and establish a meaning for Thermodynamic Transformations.

Keywords: Characteristic Functions. Thermodynamic Cube. Teaching of Thermodynamics. S-Digraph representation.

\section{INTRODUÇÃO}

As Funções Potenciais da Termodinâmica têm várias denominações, a exemplo de Funções Características, Potenciais de Massieu, Função Fundamental, Entalpia Livre, dentre outros. Planck (1897, p. 109) usou o termo "Charakteristische Funktion" para se referir às Funções Potenciais de Massieu.

Entretanto, o termo Funções Características surgiu para a Termodinâmica numa data anterior à citação de Planck, mais precisamente na segunda metade do século 19 nas publicações científicas de Massieu (1869, apud BALIAN, 2017), quando foram apresentadas duas Funções Potenciais na Representação da Entropia: Observa-se que a primeira dessas funções, denominada por Potencial de Massieu, é proporcional à Energia Livre de Helmholtz; enquanto que a segunda dessas funções apresentadas por Massieu, também conhecida como Potencial de Planck (BALIAN, 2017), é proporcional à Energia Livre de Gibbs. Segundo Balian (2015, p. 2), “As funções características de Massieu, redescobertas por Max Planck (1897) e referidas sob vários nomes, só foram apresentadas em livros didáticos recentemente por Hebert Callen (1960)".

Segundo Callen (1960, p. 101),

O conceito de potencial termodinâmico foi criado por F. Massieu em dois pequenos ensaios publicados em 1869 no Comptes Rendus. O resultado de Massieu (1869, p. 859) foi escrever uma função por meio da qual se pode tirar todas as propriedades termodinâmicas dos corpos. Ele chamou esta função de função característica (Apud SOUZA FILHO, 1998, p. 4).

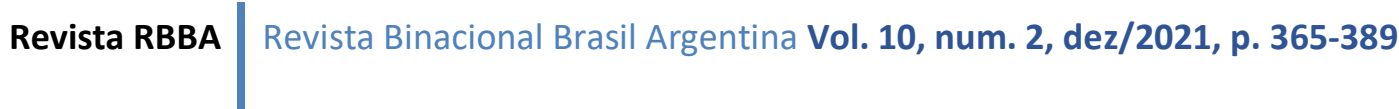


Segundo Planck (1897, p. 109), as equações e relações matemáticas do Sistema Termodinâmico, derivadas inequivocamente por diferenciação simples das propriedades termodinâmicas do sistema, devem garantir a condição de equilíbrio termodinâmico.

Historicamente, as Funções Características, na representação da Entropia, foram desenvolvidas e introduzidas na Termodinâmica por Massieu e Planck, enquanto que as Funções das Energias Potenciais Termodinâmicas foram desenvolvidas por Carnot, Mayer, Joule, Helmholtz, Clausius, Thompson, Zeuner, Maxwell, van der Waals, Gibbs, Boltzmann, Duhen, Landau dentre outros.

A contribuição de Massieu, no tocante às funções potenciais,

teve pouco impacto, talvez porque sua forma de escrever tinha caraterística tecnicista (embora ele tenha escrito em um estilo científico e abordava os avanços recentes, como a Entropia de Clausius). Mesmo atualmente, a maioria dos livros didáticos ainda o ignora, dando crédito a Gibbs pela invenção dos potenciais termodinâmicos. De fato, como é bem sabido, Gibbs [10] introduziu em 1876, sob o nome de "função fundamental" o que hoje chamamos de "Entalpia Livre" $G(T, p,\{N i\})=U-T S+P V$ para um fluido feito de moléculas $\{\mathrm{Ni}\}$ de diferentes espécies. No entanto, o próprio Gibbs havia escrito claramente em uma nota de rodapé: "Massieu parece ter sido o primeiro a resolver o problema de representar todas as propriedades de um corpo de composição invariável que estão envolvidos em processos reversíveis por meio de uma única função". De fato, a função de Gibbs pode ser considerada uma extensão da segunda função característica de Massieu para uma mistura que pode sofrer reações químicas. Da mesma forma, a "Energia Livre" $F(T, p,\{N i\})=U-T S$ introduzida em 1882 por Helmholtz [11] aparece como uma extensão para misturas da primeira função característica de Massieu. Duhem [12], que propôs denominar essas funções de "potenciais termodinâmicos", atribui apropriadamente sua ideia a Massieu, e sua introdução na termoquímica a Gibbs. Os mesmos créditos são dados por Poincaré [13], que como Duhem apresenta as funções de Massieu em sua forma modificada $H$ e $H^{\prime}$ de 1876. No entanto, o Potencial de Planck [14] não é outra coisa senão a forma original $\psi^{\prime}$ da segunda função característica de Massieu; Planck, assim como muitos outros, parece ter sido um conhecedor da obra de Massieu. O interesse conceitual dos potenciais termodinâmicos originais de Massieu $\psi \mathrm{e}$ $\psi^{\prime}$ começou a ser enfatizado apenas um século depois, por Callen [15]. O sistema composto em estudo foi isolado e analisado em subsistemas, cada um dos quais em equilíbrio térmico. (BALIAN, 2017, p. 529).

Embora as funções de Massieu-Planck estejam fortemente relacionadas às Transformadas de Legendre (DEBENEDETTI, 1986), este artigo propõe um modelo cúbico que visa simplificar o equacionamento das evoluções de um sistema sem considerar, de forma

Revista RBBA $\mid$ Revista Binacional Brasil Argentina Vol. 10, num. 2, dez/2021, p. 365-389 
explícita, as Transformadas de Lengendre. Nesse sentido, os potenciais termodinâmicos, vinculados de forma direcionada por Setas Arestas, estão distribuídos nos vértices do cubo proposto. As variáveis livres dos potenciais, localizadas nas faces desse mesmo cubo estão articuladas duas a duas por Setas Coordenadas. Tomou-se como referência o Cubo Termodinâmico na representação da Energia proposto por Correia (2021b), de forma a complementar o modelo do Quadrado Mnemônico de Born (ZHAO, 2009), do Cubo Termodinâmico de Pate (PATE, 1999), do Diagrama Termodinâmico de Venn (KERR \& MACOSKO, 2011), do Cubo de Pogliani (2018) e do Cubo Mágico de Kocik (2018). Este artigo também visa facilitar a obtenção das equações e as relações dos potenciais termodinâmicos para sistemas abertos. A energia, por unidade de temperatura, necessária para promover a transformação do potencial localizado em um vértice do cubo para o potencial localizado em outro vértice adjacente ao primeiro é proporcional ao produto das variáveis articuladas pela Seta Coordenada que estiver na direção paralela à direção da Seta Aresta que vincula esses dois potencias.

O modelo ora apresentado estabelece uma interdependência dos potenciais termodinâmicos de Massieu para Sistemas Simples que, segundo Callen (1985), os Sistemas Simples

são macroscopicamente homogêneos, isotrópicos, descarregados eletricamente, e são grandes o suficiente para que os efeitos de superfície possam ser negligenciados e que não sejam influenciados por campos elétricos, magnéticos ou gravitacionais. (CALLEN, 1985, p. 9).

Na seção seguinte será apresentado o Cubo Termodinâmico proposto.

2. O Cubo Termodinâmico, as Setas Arestas, as Setas Coordenadas, os Potenciais Termodinâmicos e as Variáveis Conjugadas

\subsection{Apresentação do Cubo Termodinâmico para as Funções Características de Massieu}

Cada um dos Potenciais $\left(S, \psi, \psi^{\prime}, \varphi, F, \phi, H\right.$ e 0$)$ ocupa um dos vértices do Cubo Termodinâmico.

O sistema de eixos ortogonais da Figura 1 tem a mesma estrutura das Setas Coordenadas e das Setas Arestas do cubo proposto por Correia (2021b). Ao substituir as energias potenciais

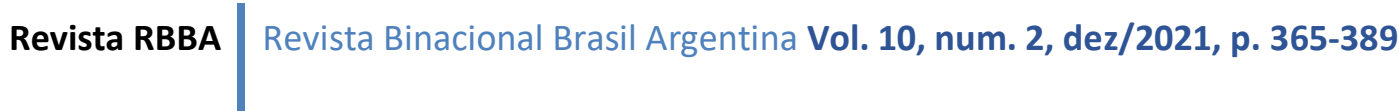


nos vértices do cubo de Correia (2021b) por potenciais termodinâmicos, foi necessário definir outras variáveis livres nas extremidades dos eixos coordenados.

Denominações dos Potenciais Termodinâmicos da Figura 1:

$S \quad$ Entropia;

$\psi \quad$ Primeira Função Característica de Massieu;

$\psi^{\prime} \quad$ Segunda Função Característica de Massieu ou Potencial de Planck;

$\varphi \quad$ Potencial anônimo;

F Potencial anônimo (Nome sugerido: "Potencial de Boltzmann");

$\phi \quad$ Potencial anônimo;

H Potencial anônimo;

$0 \quad$ Potencial Nulo.

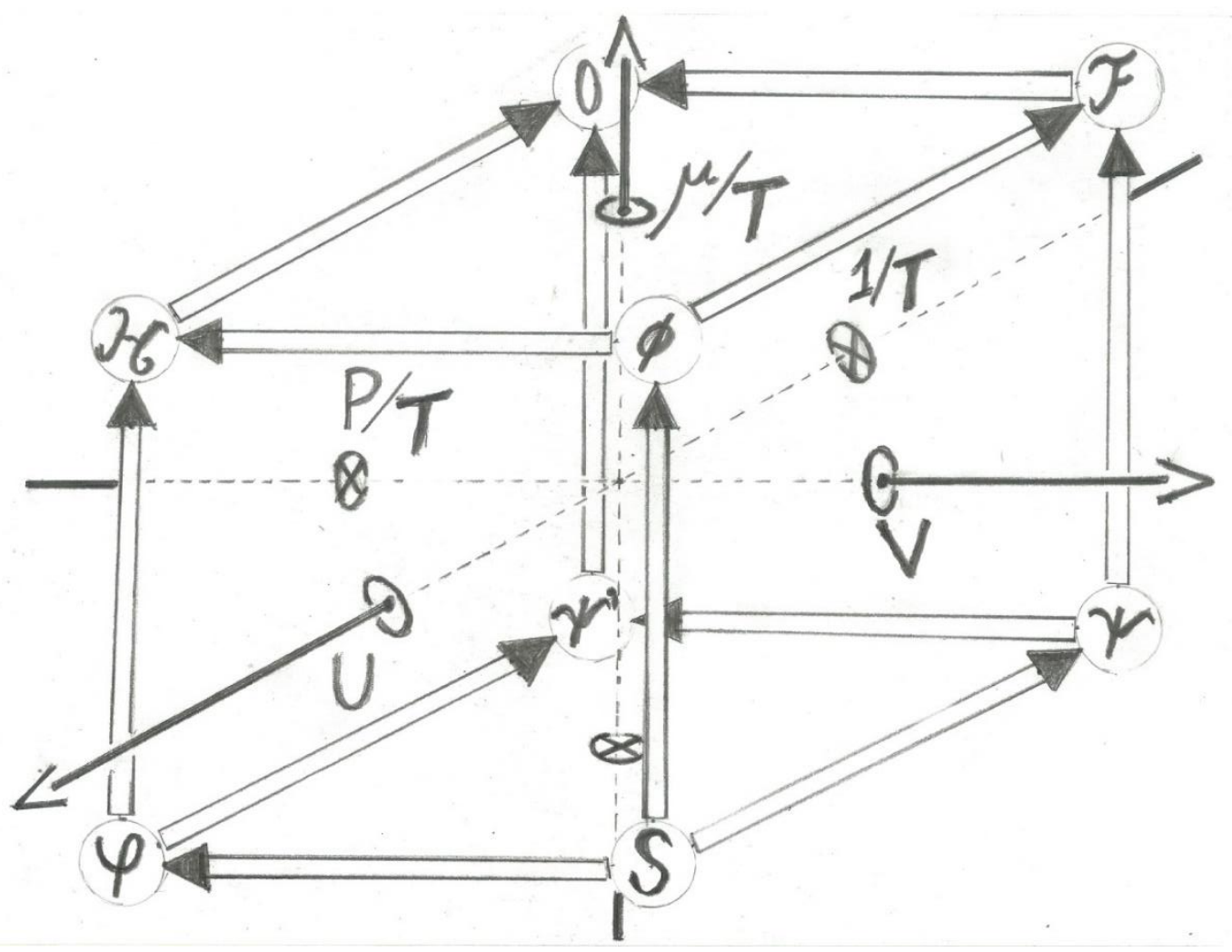

Figura 1. Cubo Termodinâmico para os Potenciais de Massieu.

Nas duas subseções seguintes serão definidas as Setas Aresta e as Setas Coordenadas do Cubo Termodinâmico.

Revista RBBA $\mid$ Revista Binacional Brasil Argentina Vol. 10, num. 2, dez/2021, p. 365-389 
CARACTERÍSTICAS DE MASSIEU

\subsection{Setas Arestas}

A Seta Aresta para o cubo $S$-digráfico é uma denominação genérica para cada um dos vetores localizados nas arestas do cubo da Figura 1. Esses eixos orientados visam denotar os sentidos das transformações dos potenciais das origens de cada uma dessas setas aos potenciais localizados nas extremidades de cada uma dessas mesmas setas.

\subsection{Setas Coordenadas}

As setas Coordenadas são os eixos orientados que se cruzam no centro do Cubo Termodinâmico. A grandeza física relativa ao produto dessas duas variáveis conjugadas por uma mesma Seta Coordenada tem a dimensão de Energia Potencial dividida pela Temperatura Absoluta. Essa energia, por unidade de temperatura, adicionada ou subtraída do sistema, é decorrente do produto das variáveis livres vinculadas pela Seta Aresta, promove a transformação dos potenciais termodinâmicos vinculados pela Seta Coordenada que estiver na mesma direção da Seta Aresta.

A disposição dessas variáveis livres neste sistema ortogonal, com base na Figura 1, é a seguinte:

- No eixo $\frac{\overrightarrow{1} T}{T}$, o inverso da temperatura absoluta $\left(\frac{1}{T}\right)$ está localizado na origem (ladocruz do cubo), enquanto que a Energia Interna $(U)$ está localizada na extremidade (lado-ponto do cubo) deste mesmo eixo;

- No eixo $\frac{\vec{P}}{T}$, a razão da pressão pela temperatura absoluta $\left(\frac{P}{T}\right)$ está localizada na origem (lado-cruz do cubo), enquanto que o Volume (V) está localizado na extremidade (lado-ponto do cubo) deste mesmo eixo;

- No eixo $\overrightarrow{N \frac{\mu}{T}}$, o número de partículas $(N)$ está localizado na origem (lado-cruz do cubo), enquanto que a razão do potencial químico pela temperatura absoluta $\left(\frac{\mu}{T}\right)$ está localizada na extremidade (lado-ponto do cubo) deste mesmo eixo.

Note que a face do cubo que contém o lado-cruz é paralela à face cubo que contém o lado-ponto.

Cada uma das doze Setas Arestas vincula, de forma direcionada, um par dentre os oito potenciais termodinâmicos de Massieu.

O cubo ora proposto é composto por um conjunto de dois grafos completos e desconexos, em que a Entropia representa o "potencial fonte", enquanto que o Potencial Nulo representa o "potencial sumidouro".

Revista RBBA Revista Binacional Brasil Argentina Vol. 10, num. 2, dez/2021, p. 365-389 
As Regras de Simetria que regem os sinais a serem aplicadas nas Equações de Estado dos Potencias Termodinâmicos estão apresentadas na seção que se segue.

\subsection{As Equações de Estado para as Funções Características de Massieu}

Os Potenciais Termodinâmicos podem ser combinados convenientemente com a utilização das Setas Arestas e das Setas Coordenadas, cujas equações matemáticas apresentam a seguinte estrutura:

$$
x_{l}=x_{i} \pm x_{j} x_{k},(j=k)
$$

A Equação (01), apresentada aqui de forma alternativa à obtida pela Transformada de Legendre, indica que um sistema pode evoluir do estado inicial $x_{i}$, localizado na origem da Seta Aresta, ao estado final $x_{l}$, localizado na extremidade dessa mesma seta. O termo $x_{j} x_{k}$ $\left(\frac{U}{T}, \frac{P V}{T}\right.$, ou $\left.\frac{\mu N}{T}\right)$ representa a energia de estado (Interna, Mecânica ou Química) dividida pela Temperatura Absoluta, cujo resultado é proveniente do produto entre a grandeza intensiva $x_{j}$ $\left(\frac{1}{T}, \frac{P}{T}\right.$, ou $\left.\frac{\mu}{T}\right)$ e a grandeza extensiva $x_{k}(U, V$ ou $N)$ que estão articuladas pela Seta Coordenada paralela à Seta Aresta. A condição ${ }_{j=k}$ ) na Equação (01) é a garantia de que a ordem da grandeza extensiva do produto $x_{j} x_{k}$ seja da mesma ordem da grandeza intensiva.

As regras dos sinais das equações de estado adotadas neste modelo, que descrevem as transformações dos potenciais termodinâmicos, são apresentadas na seção que se segue.

\subsection{As Regras de Sinais das Equações de Estado}

As regras de sinais para a Equação (01) são as mesmas apresentadas por Correia (2021b). A primeira regra é:

- “ $x_{l}$ " e " $x_{i}$ ” são sempre precedidos por sinais positivos.

A segunda regra é:

- O produto " $x_{j} x_{k}$ " será precedido de sinal positivo se a Seta Coordenada que liga $x_{j}$ a $x_{k}$ estiver no mesmo sentido da Seta Aresta que liga " $x_{l}$ " a " $x_{i}$ ";

- O produto " $x_{j} x_{k}$ " será precedido de sinal negativo se a Seta Coordenada que liga $x_{j}$ a $x_{k}$ estiver no sentido contrário ao da Seta Aresta que liga " $x_{l}$ " a " $x_{i}$ ".

Revista RBBA $\mid$ Revista Binacional Brasil Argentina Vol. 10, num. 2, dez/2021, p. 365-389 
Segue abaixo as doze Equações de Estado dos Potenciais Termodinâmicos podem ser obtidas por meio destas regras:

$$
\begin{aligned}
& \phi=S+\frac{\mu N}{T} \\
& 0=\psi^{\prime}+\frac{\mu N}{T} \\
& \mathcal{H}=\varphi+\frac{\mu N}{T} \\
& F=\psi+\frac{\mu N}{T} \\
& \mathcal{H}=\phi-\frac{P V}{T} \\
& 0=F-\frac{P V}{T} \\
& \psi^{\prime}=\psi-\frac{P V}{T} \\
& \varphi=S-\frac{P V}{T} \\
& 0=\mathcal{H}-\frac{U}{T} \\
& F=\phi-\frac{U}{T} \\
& \psi=S-\frac{U}{T} \\
& \psi^{\prime}=\varphi-\frac{U}{T}
\end{aligned}
$$

\subsection{As Funções Características de Massieu e suas Variáveis Livres}

Cada um dos oito Potenciais Termodinâmicos de Massieu possui três variáveis livres, cujas descrições serão apresentadas nesta seção. Cada uma das três variáveis livres de cada um desses potenciais está localizada no centro de uma das três faces que formam o vértice do Cubo Termodinâmico. Ou seja:

$$
\begin{aligned}
& S=f(U, V, N) ; \\
& \psi=f\left(\frac{1}{T}, V, N\right) ; \\
& \varphi=f\left(U, \frac{P}{T}, N\right) ; \\
& \psi^{\prime}=f\left(\frac{1}{T}, \frac{P}{T}, N\right) ; \\
& \phi=f\left(U, V, \frac{\mu}{T}\right) ;
\end{aligned}
$$

Revista RBBA $\quad$ Revista Binacional Brasil Argentina Vol. 10, num. 2, dez/2021, p. 365-389 


$$
\begin{aligned}
& F=f\left(\frac{1}{T}, V, \frac{\mu}{T}\right) ; \\
& \mathcal{H}=f\left(U, \frac{P}{T}, \frac{\mu}{T}\right) ; \\
& 0=f\left(\frac{1}{T}, \frac{P}{T}, \frac{\mu}{T}\right) .
\end{aligned}
$$

$\mathrm{Na}$ seção seguinte será apresentada a diferencial de cada um dos Potenciais Termodinâmicos.

\subsection{A Equação Diferencial Exata das Funções Características de Massieu}

A variação infinitesimal $(d)$ do potencial $[\phi(x, y, z)]$ localizado em cada aresta deste cubo será descrita pela diferencial exata, truncada em primeira ordem:

$$
d \phi(x, y, z)= \pm X d x \pm Y d y \pm Z d z
$$

Considerando que a intersecção de três faces de um cubo dá origem a um vértice e que em cada vértice do cubo corresponde à localização de um potencial termodinâmico $\phi(x, y, z)$, temse:

- $\phi(x, y, z)$ é o potencial termodinâmico localizado no vértice do cubo em análise;

- $\quad x(y$ ou $z)$ é a variável livre localizada no centro de uma das três faces;

- $X(Y$ ou $Z)$ é a variável livre localizada no centro da face paralela à face que contém $x(y$ ou $z)$.

Essa equação diferencial exata para os Potenciais Termodinâmicos é denominada por Forma Diferencial de Gibbs.

As derivadas de um potencial $\phi\left(x_{i}, x_{j}, x_{k}\right)$ em relação a uma de suas variáveis livres $x_{i}$, $x_{j}$ ou $x_{k}$ são descritas na forma que se segue:

$$
\begin{aligned}
& \left.\frac{\partial\left[\phi\left(x_{i}, x_{j}, x_{k}\right)\right]}{\partial x_{i}}\right|_{x_{j}, x_{k}}= \pm X ; \\
& \left.\frac{\partial\left[\phi\left(x_{i}, x_{j}, x_{k}\right)\right]}{\partial x_{j}}\right|_{x_{i}, x_{k}}= \pm Y ; \\
& \left.\frac{\partial\left[\phi\left(x_{i}, x_{j}, x_{k}\right)\right]}{\partial x_{k}}\right|_{x_{i}, x_{j}}= \pm Z .
\end{aligned}
$$


Os sinais (positivo e negativo) que precedem as variáveis livres $X, Y$ ou $Z$ obedecem à terceira regra de sinal, cujo texto é:

- A variável $X(Y$ ou $Z)$ será precedida de sinal positivo se ela estiver localizada num lado-cruz;

- A variável $X(Y$ ou $Z$ ) será precedida de sinal negativo se ela estiver localizada num lado-ponto.

Considerando esta regra de sinais, as equações diferenciais para cada um dos Potenciais Termodinâmicos de Massieu podem ser obtidas a partir da Equação (22).

A equação diferencial para a Entropia $S=f(U, V, N)$ é

$$
\begin{gathered}
d S=\left(+\frac{1}{T}\right) d U+\left(+\frac{P}{T}\right) d V+\left(-\frac{\mu}{T}\right) d N \\
d S=\frac{1}{T} d U+\frac{P}{T} d V-\frac{\mu}{T} d N .
\end{gathered}
$$

A equação diferencial para a Primeira Função Característica de Massieu $\psi=f\left(\frac{1}{T}, V, N\right)$ é

$$
\begin{array}{r}
d \psi=(-U) d\left(\frac{1}{T}\right)+\left(+\frac{P}{T}\right) d V+\left(-\frac{\mu}{T}\right) d N \\
d \psi=-U d\left(\frac{1}{T}\right)+\frac{P}{T} d V-\frac{\mu}{T} d N .
\end{array}
$$

A equação diferencial para a Segunda Função Característica de Massieu $\psi^{\prime}=f\left(\frac{1}{T}, \frac{P}{T}, N\right)$ é

$$
\begin{array}{r}
d \psi^{\prime}=(-U) d\left(\frac{1}{T}\right)+(-V) d\left(\frac{P}{T}\right)+\left(-\frac{\mu}{T}\right) d N \\
d \psi^{\prime}=-U d\left(\frac{1}{T}\right)-V d\left(\frac{P}{T}\right)-\frac{\mu}{T} d N .
\end{array}
$$

A equação diferencial para o Potencial anônimo $\varphi=f\left(U, \frac{P}{T}, N\right)$ é

$$
\begin{gathered}
\left.d \varphi=\left(+\frac{1}{T}\right)\right) d(U)+(-V) d\left(\frac{P}{T}\right)+\left(-\frac{\mu}{T}\right) d(N) \\
d \varphi=\frac{1}{T} d U-\frac{P}{T} d V-\frac{\mu}{T} d N .
\end{gathered}
$$

A equação diferencial para o Potencial Anônimo $\phi=f\left(U, V, \frac{\mu}{T}\right)$ é

$$
\begin{gathered}
d \phi=\left(+\frac{1}{T}\right) d U+\left(+\frac{P}{T}\right) d V+(+N) d\left(\frac{\mu}{T}\right) \\
d \phi=\frac{1}{T} d U+\frac{P}{T} d V+N d\left(\frac{\mu}{T}\right) .
\end{gathered}
$$

A equação diferencial para o Potencial Anônimo ("Potencial de Boltzmann") $\mathcal{F}=f\left(\frac{1}{T}, V, \frac{\mu}{T}\right)$ é 


$$
\begin{gathered}
d \mathcal{F}=(-U) d)\left(\frac{1}{T}\right)+\left(+\frac{P}{T}\right) d(V)+(+N) d\left(\frac{\mu}{T}\right) \\
d \mathcal{F}=-U d\left(\frac{1}{T}\right)+\frac{P}{T} d V+N d\left(\frac{\mu}{T}\right)
\end{gathered}
$$

A equação diferencial para o Potencial Anônimo $\mathcal{H}=f\left(U, \frac{P}{T}, \frac{\mu}{T}\right)$ é;

$$
\begin{gathered}
\left.d \mathcal{H}=\left(+\frac{1}{T}\right) d U+V\right) d\left(\frac{P}{T}\right)+(-N) d\left(\frac{\mu}{T}\right) \\
d \mathcal{H}=\frac{1}{T} d U+\frac{P}{T} d V-N d\left(\frac{\mu}{T}\right) .
\end{gathered}
$$

A equação diferencial para o Potencial Nulo $0=f\left(\frac{1}{T}, \frac{P}{T}, \frac{\mu}{T}\right)$ é

Como $d 0=0$, então

$$
\begin{gathered}
d 0=(-U) d\left(\frac{1}{T}\right)+(-V) d\left(\frac{P}{T}\right)+(+N) d\left(\frac{\mu}{T}\right) \\
d 0=-U d\left(\frac{1}{T}\right)-V d\left(\frac{P}{T}\right)+N d\left(\frac{\mu}{T}\right)
\end{gathered}
$$

$$
\begin{gathered}
O=(-U) d\left(\frac{1}{T}\right)+(-V) d\left(\frac{P}{T}\right)+(+N) d\left(\frac{\mu}{T}\right) \\
O=-U d\left(\frac{1}{T}\right)-V d\left(\frac{P}{T}\right)+N d\left(\frac{\mu}{T}\right) .
\end{gathered}
$$

\subsection{A Dedução da Equação de Euler para os Potenciais de Massieu}

Vimos apresentar nesta seção a demonstração da Equação de Euler da Termodinâmica, conforme Correia (2021b). Visando simplificar a demonstração desta relação matemática, considere que a função $\phi\left(x_{1}, x_{2}\right)$ dependa apenas das duas variáveis $x_{1}$ e $x_{2}$.

Essa grandeza $\phi\left(x_{1}, x_{2}\right)$ será homogênea em primeira ordem se

$$
\phi\left(\lambda x_{1}, \lambda x_{2}\right)=\lambda \phi\left(x_{1}, x_{2}\right) .
$$

Substituindo $\lambda x_{1}$ por $u_{1}$ e $\lambda x_{2}$ por $u_{2}$ temos

$$
\phi\left(\lambda x_{1}, \lambda x_{2}\right)=\phi\left(u_{1}, u_{2}\right)
$$

Derivando $\phi\left(\lambda x_{1}, \lambda x_{2}\right)$ em relação a $\lambda$, pela Regra da Cadeia, temos

$$
\frac{\partial\left[\phi\left(\lambda x_{1}, \lambda x_{2}\right)\right]}{\partial \lambda}=\frac{\partial\left[\phi\left(u_{1}, u_{2}\right)\right]}{\partial u_{1}} \frac{\partial u_{1}}{\partial \lambda}+\frac{\partial\left[\phi\left(u_{1}, u_{2}\right)\right]}{\partial u_{2}} \frac{\partial u_{2}}{\partial \lambda} .
$$

Mas como $\frac{\partial u_{1}}{\partial \lambda}=x_{1}, \frac{\partial u_{2}}{\partial \lambda}=x_{2} \mathrm{e} \frac{\partial\left[\phi\left(\lambda x_{1}, \lambda x_{2}\right)\right]}{\partial \lambda}=\phi\left(x_{1}, x_{2}\right)$, a expressão da derivada parcial da grandeza $\phi\left(\lambda x_{1}, \lambda x_{2}\right)$ em relação a $\lambda$ fica representada pela relação

$$
\phi\left(x_{1}, x_{2}\right)=\frac{\partial\left[\phi\left(u_{1}, u_{2}\right)\right]}{\partial u_{1}} x_{1}+\frac{\partial\left[\phi\left(u_{1}, u_{2}\right)\right]}{\partial u_{2}} x_{2} .
$$

Mas, como $u_{1}=\lambda x_{1}$ e $u_{2}=\lambda x_{2}$, obtém-se

$$
\phi\left(x_{1}, x_{2}\right)=\frac{\partial\left[\phi\left(\lambda x_{1}, \lambda x_{2}\right)\right]}{\partial\left(\lambda x_{1}\right)} x_{1}+\frac{\partial\left[\phi\left(\lambda x_{1}, \lambda x_{2}\right)\right]}{\partial\left(\lambda x_{2}\right)} x_{2} .
$$

$\mathrm{E}, \operatorname{como} \phi\left(x_{1}, x_{2}\right)$ é uma função homogênea, então

Revista RBBA $\mid$ Revista Binacional Brasil Argentina Vol. 10, num. 2, dez/2021, p. 365-389 


$$
\phi\left(x_{1}, x_{2}\right)=\frac{\lambda \partial\left[\phi\left(x_{1}, x_{2}\right)\right]}{\lambda \partial\left(x_{1}\right)} x_{1}+\frac{\lambda \partial\left[\phi\left(x_{1}, x_{2}\right)\right]}{\lambda \partial\left(x_{2}\right)} x_{2}
$$

Finalmente

$$
\phi\left(x_{1}, x_{2}\right)=\frac{\partial\left[\phi\left(x_{1}, x_{2}\right)\right]}{\partial x_{1}} x_{1}+\frac{\partial\left[\phi\left(x_{1}, x_{2}\right)\right]}{\partial x_{2}} x_{2} .
$$

Com base na Equação (41), cada uma das equações das funções características pode ser obtida de forma alternativa à técnica em que se utilizam as Transformadas de Legendre.

Como exemplo da aplicação deste método, considere o potencial termodinâmico denominado por Entropia $(S)$ com suas variáveis livres $(U, V$ e $N)$.

Considerando $S=f(U, V, N)$ :

$$
S=\frac{\partial S}{\partial U} U+\frac{\partial S}{\partial V} V+\frac{\partial S}{\partial N} N .
$$

Mas, efetuando operações no cubo termodinâmico e aplicando a terceira regra de sinais apresentadas acima temos:

$$
\left.\begin{array}{l}
\frac{\partial S}{\partial U}=\frac{1}{T} \\
\frac{\partial S}{\partial V}=\frac{P}{T} \\
\frac{\partial S}{\partial N}=-\frac{\mu}{T}
\end{array}\right\} .
$$

Com isso

$$
S=\frac{1}{T} U+\frac{P}{T} V-\frac{\mu}{T} N .
$$

A Equação (44), na representação da Entropia, é equivalente à "Relação de Euler da Termodinâmica" na representação da Energia (CORREIA, 2021b, Equação 052), cuja quantidade é proveniente da divisão pela Temperatura Absoluta do resultado da subtração das energias Mecânica e Química da Energia Interna.

A diferencial da Equação (44) dá origem a outra equação fundamental da Termodinâmica, cuja demonstração está na seção a seguir.

\subsection{A Dedução da Relação de Gibbs-Duhen na Representação da Entropia}

Diferenciando a Equação 44, tem-se:

$$
d S=U d\left(\frac{1}{T}\right)+\left(\frac{1}{T}\right) d U+V d\left(\frac{P}{T}\right)+\left(+\frac{P}{T}\right) d V+N d\left(-\frac{\mu}{T}\right)+\left(-\frac{\mu}{T}\right) d N .
$$

Ou ainda

Revista RBBA $\quad$ Revista Binacional Brasil Argentina Vol. 10, num. 2, dez/2021, p. 365-389 


$$
d S=\frac{1}{T} d U+\frac{P}{T} d V-\frac{\mu}{T} d N+\left[U d\left(\frac{1}{T}\right)+V d\left(\frac{P}{T}\right)-N d\left(\frac{\mu}{T}\right)\right] .
$$

Mas, de acordo com a Equação (34), a parcela entre colchetes na Equação (46) é identicamente nula. Com isso

$$
U d\left(\frac{1}{T}\right)+V d\left(\frac{P}{T}\right)-N d\left(\frac{\mu}{T}\right)=0
$$

A Equação (47) é a mesma Equação (34), apesar delas terem sido obtidas por procedimentos distintos.

A Equação (34), denominada por Relação de Gibbs-Duhen na representação da Entropia, depende apenas das variáveis intensivas $\left(\frac{1}{T}, \frac{P}{T}, \frac{\mu}{T}\right)$ (), enquanto que a Equação (44), denominada por Forma Diferencial da Relação de Euler da Termodinâmica na representação da Entropia, depende das variáveis extensivas $(U, V, N)$.

$\mathrm{Na}$ seção seguinte serão apresentados os Princípios de Conservação associados às Funções Características de Massieu.

\section{Os Princípios de Conservação das Funções Características de Massieu}

Nas duas subseções seguintes serão enunciados dois princípios de conservação associados aos Potenciais Termodinâmicos de Massieu.

\subsection{O Primeiro Princípio de Conservação dos Potenciais Termodinâmicos}

A soma dos potenciais localizados nos extremos de uma das diagonais de uma face do cubo é igual à soma dos potenciais localizados nos extremos da outra diagonal dessa mesma face. Desta regra de simetria podem ser obtidas as seguintes relações:

$$
\begin{aligned}
& S+\psi^{\prime}=\psi+\varphi \\
& \phi+0=\mathcal{F}+\mathcal{H} ; \\
& \psi+\phi=S+\mathcal{F} \\
& \psi^{\prime}+\mathcal{H}=\varphi+0 \\
& S+\mathcal{H}=\varphi+\phi
\end{aligned}
$$

Revista RBBA $\quad$ Revista Binacional Brasil Argentina Vol. 10, num. 2, dez/2021, p. 365-389 


$$
\psi+0=\psi^{\prime}+\mathcal{F} .
$$

\subsection{O Segundo Princípio de Conservação dos Potenciais Termodinâmicos}

A soma dos potenciais nos extremos a uma diagonal principal deste Cubo Termodinâmico é igual à Entropia $S$. Dessa regra podemos obter as seguintes relações:

$$
\begin{aligned}
& 0+S=S \\
& \varphi+\mathcal{F}=S \\
& \phi+\psi^{\prime}=S \\
& \psi+\mathcal{H}=S
\end{aligned}
$$

A partir do Cubo Termodinâmico serão apresentadas, na seção que se segue, algumas operações de simetria de forma alternativa às Transformadas de Legendre, de modo a obter as Relações de Gibbs e obter as Identidades de Maxwell na representação da Entropia.

\section{As Relações de Gibbs para os Potenciais de Massieu}

As Relações de Gibbs da Termodinâmica também podem ser obtidas a partir de operações do Cubo Termodinâmico, ao se utilizar a seguinte Regra de Simetria:

$$
\left.\frac{\partial}{\partial x_{i}}\left[\phi\left(x_{i}, x_{j}, x_{k}\right)\right]\right|_{x_{j}, x_{k}}= \pm x_{l}
$$

Embora esta regra de sinais já tenha sido apresentada na seção 2.5, seguem as denominações com destaque para as variáveis livres da Equação (58):

- $\phi\left(x_{i}, x_{j}, x_{k}\right)$ é um potencial termodinâmico localizado em um dos vértices do cubo, que depende das variáveis $x_{i}, x_{j}$ e $x_{k}$;

- $x_{j}$ e $x_{k}$, são as variáveis livres mantidas constantes, enquanto que o potencial $\phi\left(x_{i}, x_{j}, x_{k}\right)$ estiver sendo derivado em relação à variável livre $x_{i}$;

- $x_{l}$ é a variável livre localizada no outro extremo da Seta Coordenada limitada pelos dois extremos $x_{i}$ e $x_{l}$.

Destas regras, na representação da Entropia, pode ser obtido um conjunto de relações equivalentes às Relações de Gibbs que foram obtidas na representação da Energia (CORREIA, 2021b):

Revista RBBA $\quad$ Revista Binacional Brasil Argentina Vol. 10, num. 2, dez/2021, p. 365-389 


$$
\begin{aligned}
& \left.\frac{\partial \mathcal{H}}{\partial\left(\frac{P}{T}\right)}\right|_{U, \frac{\mu}{T}}=\left.\frac{\partial 0}{\partial\left(\frac{P}{T}\right)}\right|_{\frac{1}{T}, \frac{\mu}{T}}=\left.\frac{\partial \varphi}{\partial\left(\frac{P}{T}\right)}\right|_{U, N}=\left.\frac{\partial \psi \prime}{\partial\left(\frac{P}{T}\right)}\right|_{\frac{1}{T}, N}=-V ; \\
& \left.\frac{\partial \mathcal{F}}{\partial\left(\frac{1}{T}\right)}\right|_{V, \frac{\mu}{T}}=\left.\frac{\partial 0}{\partial\left(\frac{1}{T}\right)}\right|_{\frac{P}{T}, \frac{\mu}{T}}=\left.\frac{\partial \psi^{\prime}}{\partial\left(\frac{1}{T}\right)}\right|_{\frac{P}{T}, N}=\left.\frac{\partial \psi}{\partial\left(\frac{1}{T}\right)}\right|_{V, N}=-U ; \\
& \left.\frac{\partial 0}{\partial\left(\frac{\mu}{T}\right)}\right|_{\frac{1}{T}, \frac{\mu}{T}}=\left.\frac{\partial \mathcal{H}}{\partial\left(\frac{\mu}{T}\right)}\right|_{\bar{T}, U}=\left.\frac{\partial \phi}{\partial\left(\frac{\mu}{T}\right)}\right|_{U, V}=\left.\frac{\partial \mathcal{F}}{\partial\left(\frac{\mu}{T}\right)}\right|_{V, \frac{1}{T}}=N ; \\
& \left.\frac{\partial \psi}{\partial N}\right|_{\frac{1}{T}, V}=\left.\frac{\partial S}{\partial N}\right|_{U, V}=\left.\frac{\partial \varphi}{\partial N}\right|_{U, \frac{P}{T}}=\left.\frac{\partial \psi^{\prime}}{\partial N}\right|_{\frac{P}{T}, \frac{1}{T}}=-\frac{\mu}{T} ; \\
& \left.\frac{\partial S}{\partial V}\right|_{U, N}=\left.\frac{\partial \psi}{\partial V}\right|_{\frac{1}{T}, N}=\left.\frac{\partial \mathcal{F}}{\partial V}\right|_{\frac{1}{T}, \frac{\mu}{T}}=\left.\frac{\partial \phi}{\partial V}\right|_{\frac{\mu}{T}, U}=\frac{P}{T} ; \\
& \left.\frac{\partial S}{\partial U}\right|_{N, V}=\left.\frac{\partial \phi}{\partial U}\right|_{V, \frac{\mu}{T}}=\left.\frac{\partial \mathcal{H}}{\partial U}\right|_{\frac{P}{T}, \frac{\mu}{T}}=\left.\frac{\partial \varphi}{\partial U}\right|_{\frac{P}{T}, N}=\frac{1}{T} .
\end{aligned}
$$

\subsection{As Identidades de Maxwell para os Potenciais de Massieu}

Cada uma das Identidades de Maxwell, denominada por Relações de Maxwell, será obtida a partir do Teorema de Schwartz e das Relações de Gibbs:

$$
\begin{aligned}
& \text { - }\left.\frac{\partial}{\partial x_{i}}\left(\frac{\partial}{\partial x_{j}}\right)\left[\phi\left(x_{i}, x_{j}, x_{k}\right)\right]\right|_{x_{k}}=\left.\frac{\partial}{\partial x_{j}}\left(\frac{\partial}{\partial x_{i}}\right)\left[\phi\left(x_{i}, x_{j}, x_{k}\right)\right]\right|_{x_{k}} ; \\
& \text { - }\left.\frac{\partial}{\partial x_{j}}\left(\frac{\partial}{\partial x_{k}}\right)\left[\phi\left(x_{i}, x_{j}, x_{k}\right)\right]\right|_{x_{i}}=\left.\frac{\partial}{\partial x_{k}}\left(\frac{\partial}{\partial x_{j}}\right)\left[\phi\left(x_{i}, x_{j}, x_{k}\right)\right]\right|_{x_{i}} ; \\
& \text { - }\left.\frac{\partial}{\partial x_{k}}\left(\frac{\partial}{\partial x_{i}}\right)\left[\phi\left(x_{i}, x_{j}, x_{k}\right)\right]\right|_{x_{j}}=\left.\frac{\partial}{\partial x_{i}}\left(\frac{\partial}{\partial x_{k}}\right)\left[\phi\left(x_{i}, x_{j}, x_{k}\right)\right]\right|_{x_{j}}
\end{aligned}
$$

Aplicando convenientemente a Relação de Gibbs a cada uma das oito Funções Características, obtêm-se três Identidades de Maxwell para cada um dos oito potenciais termodinâmicos de Massieu. Com isso, seriam obtidas vinte e quatro Relações de Maxwell, conforme as deduções que se seguem: 


\subsubsection{A Entropia $S$}

$S=f(V, U, N)$.

(a) $\left.\frac{\partial}{\partial U}\left(\frac{\partial}{\partial V}\right) S\right|_{N}=\left.\frac{\partial}{\partial V}\left(\frac{\partial}{\partial U}\right) S\right|_{N}$;

$\left.\frac{\partial}{\partial V} S\right|_{N}=\frac{P}{T}$ e $\left.\frac{\partial}{\partial U} S\right|_{N}=\frac{1}{T}$.

$$
\left.\frac{\partial}{\partial U}\left(\frac{P}{T}\right)\right|_{N}=\left.\frac{\partial}{\partial V}\left(\frac{1}{T}\right)\right|_{N}
$$

(b) $\left.\frac{\partial}{\partial U}\left(\frac{\partial}{\partial N}\right) S\right|_{V}=\left.\frac{\partial}{\partial N}\left(\frac{\partial}{\partial U}\right) S\right|_{V}$;

$\left.\frac{\partial}{\partial N} S\right|_{V}=-\frac{\mu}{T}$ e $\left.\frac{\partial}{\partial U} S\right|_{V}=\frac{1}{T}$.

$$
\left.\frac{\partial}{\partial U}\left(\frac{\mu}{T}\right)\right|_{V}=-\left.\frac{\partial}{\partial N}\left(\frac{1}{T}\right)\right|_{V}
$$

(c) $\left.\frac{\partial}{\partial V}\left(\frac{\partial}{\partial N}\right) S\right|_{U}=\left.\frac{\partial}{\partial N}\left(\frac{\partial}{\partial V}\right) S\right|_{U}$;

$\left.\frac{\partial}{\partial N} S\right|_{U}=-\frac{\mu}{T}$ e $\left.\frac{\partial}{\partial V} S\right|_{U}=\frac{P}{T} ;$

$$
\left.\frac{\partial}{\partial V}\left(\frac{\mu}{T}\right)\right|_{U}=-\left.\frac{\partial}{\partial N}\left(\frac{P}{T}\right)\right|_{U} .
$$

\subsubsection{O Primeiro Potencial de Massieu $\psi$}

$\psi=f\left(V, N, \frac{1}{T}\right)$.

(a) $\left.\frac{\partial}{\partial\left(\frac{1}{T}\right)}\left(\frac{\partial}{\partial V}\right) \psi\right|_{N}=\left.\frac{\partial}{\partial V}\left[\frac{\partial}{\partial\left(\frac{1}{T}\right)}\right] \psi\right|_{N}$;

$\left.\frac{\partial}{\partial V} \psi\right|_{N}=\frac{P}{T}$ e $\left.\frac{\partial}{\partial\left(\frac{1}{T}\right)} \psi\right|_{N}=-U ;$

$$
\left.\frac{\partial}{\partial\left(\frac{1}{T}\right)}\left(\frac{P}{T}\right)\right|_{N}=-\left.\frac{\partial}{\partial V} U\right|_{N}
$$

(b) $\left.\frac{\partial}{\partial\left(\frac{1}{T}\right)}\left(\frac{\partial}{\partial N}\right) \psi\right|_{V}=\left.\frac{\partial}{\partial N}\left[\frac{\partial}{\partial\left(\frac{1}{T}\right)}\right] \psi\right|_{V}$;

$\left.\frac{\partial}{\partial N} \psi\right|_{V}=-\frac{\mu}{T}$ e $\left.\frac{\partial}{\partial\left(\frac{1}{T}\right)} \psi\right|_{V}=-U ;$

$$
\left.\frac{\partial}{\partial\left(\frac{1}{T}\right)}\left(\frac{\mu}{T}\right)\right|_{V}=\left.\frac{\partial}{\partial N} U\right|_{V}
$$

(c) $\left.\frac{\partial}{\partial V}\left(\frac{\partial}{\partial N}\right) \psi\right|_{\frac{1}{T}}=\left.\frac{\partial}{\partial N}\left(\frac{\partial}{\partial V}\right) \psi\right|_{\frac{1}{T}}$;

$\left.\frac{\partial}{\partial N} \psi\right|_{\frac{1}{T}}=-\frac{\mu}{T}$ e $\left.\frac{\partial}{\partial V} \psi\right|_{\frac{1}{T}}=\frac{P}{T} ;$

$$
\left.\frac{\partial}{\partial V}\left(\frac{\mu}{T}\right)\right|_{\frac{1}{T}}=-\left.\frac{\partial}{\partial N}\left(\frac{P}{T}\right)\right|_{\frac{1}{T}}
$$




\subsubsection{O Segundo Potencial de Massieu $\psi^{\prime}$}

$\psi^{\prime}=f\left(N, \frac{1}{T}, \frac{P}{T}\right)$.

(a) $\left.\frac{\partial}{\partial\left(\frac{1}{T}\right)}\left[\frac{\partial}{\partial\left(\frac{P}{T}\right)}\right] \psi^{\prime}\right|_{N}=\left.\frac{\partial}{\partial\left(\frac{P}{T}\right)}\left[\frac{\partial}{\partial\left(\frac{1}{T}\right)}\right] \psi^{\prime}\right|_{N} ;$

$\left.\frac{\partial}{\partial\left(\frac{P}{T}\right)} \psi^{\prime}\right|_{N}=-V$ e $\left.\frac{\partial}{\partial\left(\frac{1}{T}\right)} \psi^{\prime}\right|_{N}=-U$;

$\left.\frac{\partial}{\partial\left(\frac{1}{T}\right)} V\right|_{N}=\left.\frac{\partial}{\partial\left(\frac{P}{T}\right)} U\right|_{N}$

(b) $\left.\frac{\partial}{\partial N}\left[\frac{\partial}{\partial\left(\frac{P}{T}\right)}\right] \psi^{\prime}\right|_{\frac{1}{T}}=\left.\frac{\partial}{\partial\left(\frac{P}{T}\right)}\left(\frac{\partial}{\partial N}\right) \psi^{\prime}\right|_{\frac{1}{T}}$;

$\left.\frac{\partial}{\partial\left(\frac{P}{T}\right)} \psi^{\prime}\right|_{\frac{1}{T}}=-V$ e $\left.\frac{\partial}{\partial N} \psi^{\prime}\right|_{\frac{1}{T}}=-\frac{\mu}{T}$

$$
\left.\frac{\partial}{\partial N} V\right|_{\frac{1}{T}}=\left.\frac{\partial}{\partial\left(\frac{P}{T}\right)}\left(\frac{\mu}{T}\right)\right|_{\frac{1}{T}}
$$

(c) $\left.\frac{\partial}{\partial\left(\frac{1}{T}\right)}\left(\frac{\partial}{\partial N}\right) \psi^{\prime}\right|_{\bar{T}}=\left.\frac{\partial}{\partial N}\left[\frac{\partial}{\partial\left(\frac{1}{T}\right)}\right] \psi^{\prime}\right|_{\bar{T}} ;$

$\left.\frac{\partial}{\partial N} \psi^{\prime}\right|_{\bar{T}}=-\frac{\mu}{T}$ e $\left.\frac{\partial}{\partial\left(\frac{1}{T}\right)} \psi^{\prime}\right|_{\frac{P}{T}}=-U ;$

$$
\left.\frac{\partial}{\partial\left(\frac{1}{T}\right)}\left(\frac{\mu}{T}\right)\right|_{\bar{T}}=\left.\frac{\partial}{\partial N} U\right|_{\bar{T}} .
$$

\subsubsection{O potencial Anônimo $\varphi$}

$\varphi=f\left(\frac{P}{T}, N, U\right)$.

(a) $\left.\frac{\partial}{\partial U}\left[\frac{\partial}{\partial\left(\frac{P}{T}\right)}\right] \varphi\right|_{N}=\left.\frac{\partial}{\partial\left(\frac{P}{T}\right)}\left(\frac{\partial}{\partial U}\right) \varphi\right|_{N}$;

$\left.\frac{\partial}{\partial\left(\frac{\mathrm{P}}{\mathrm{T}}\right)} \varphi\right|_{N}=-V$ e $\left.\frac{\partial}{\partial U} \varphi\right|_{N}=\frac{1}{T} ;$

$$
\left.\frac{\partial}{\partial U} V\right|_{N}=-\left.\frac{\partial}{\partial\left(\frac{P}{T}\right)}\left(\frac{1}{T}\right)\right|_{N}
$$

(b) $\left.\frac{\partial}{\partial U}\left(\frac{\partial}{\partial N}\right) \varphi\right|_{\bar{T}}=\left.\frac{\partial}{\partial N}\left(\frac{\partial}{\partial U}\right) \varphi\right|_{\frac{P}{T}} ;$

$\left.\frac{\partial}{\partial N} \varphi\right|_{\bar{T}}=-\frac{\mu}{T}$ e $\left.\frac{\partial}{\partial U} \varphi\right|_{\frac{P}{T}}=\frac{1}{T} ;$

$$
\left.\frac{\partial}{\partial U}\left(\frac{\mu}{T}\right)\right|_{\frac{P}{T}}=-\left.\frac{\partial}{\partial N}\left(\frac{1}{T}\right)\right|_{\frac{P}{T}}
$$


(c) $\left.\frac{\partial}{\partial\left(\frac{P}{T}\right)}\left(\frac{\partial}{\partial N}\right) \varphi\right|_{U}=\left.\frac{\partial}{\partial N}\left[\frac{\partial}{\partial\left(\frac{P}{T}\right)}\right] \varphi\right|_{U}$;

$\left.\frac{\partial}{\partial N} \varphi\right|_{U}=-\frac{\mu}{T}$ e $\left.\frac{\partial}{\partial\left(\frac{P}{T}\right)} \varphi\right|_{U}=-V ;$

$$
\left.\frac{\partial}{\partial\left(\frac{P}{T}\right)}\left(\frac{\mu}{T}\right)\right|_{U}=\left.\frac{\partial}{\partial N} V\right|_{U}
$$

\subsubsection{O Potencial Anônimo $\mathcal{H}$}

$\mathcal{H}=f\left(\frac{\mu}{T}, \frac{P}{T}, U\right)$.

(a) $\left.\frac{\partial}{\partial\left(\frac{\mu}{T}\right)}\left[\frac{\partial}{\partial\left(\frac{P}{T}\right)}\right] \mathcal{H}\right|_{U}=\left.\frac{\partial}{\partial\left(\frac{P}{T}\right)}\left[\frac{\partial}{\partial\left(\frac{\mu}{T}\right)}\right] \mathcal{H}\right|_{U}$;

$\left.\frac{\partial}{\partial\left(\frac{P}{T}\right)} \mathcal{H}\right|_{U}=-V$ e $\left.\frac{\partial}{\partial\left(\frac{\mu}{T}\right)} \mathcal{H}\right|_{U}=N$;

$\left.\frac{\partial}{\partial\left(\frac{\mu}{T}\right)} V\right|_{U}=-\left.\frac{\partial}{\partial\left(\frac{P}{T}\right)} N\right|_{U}$

(b) $\left.\frac{\partial}{\partial\left(\frac{\mu}{T}\right)}\left(\frac{\partial}{\partial U}\right) \mathcal{H}\right|_{\bar{T}}=\left.\frac{\partial}{\partial U}\left[\frac{\partial}{\partial\left(\frac{\mu}{T}\right)}\right] \mathcal{H}\right|_{\bar{T}}$;

$\left.\frac{\partial}{\partial U} \mathcal{H}\right|_{\bar{T}}=\frac{1}{T}$ e $\left.\frac{\partial}{\partial\left(\frac{\mu}{T}\right)} \mathcal{H}\right|_{\bar{T}}=N ;$

$$
\left.\frac{\partial}{\partial\left(\frac{\mu}{T}\right)}\left(\frac{1}{T}\right)\right|_{\frac{P}{T}}=\left.\frac{\partial}{\partial U} N\right|_{\frac{P}{T}} .
$$

(c) $\left.\frac{\partial}{\partial\left(\frac{P}{T}\right)}\left(\frac{\partial}{\partial U}\right) \mathcal{H}\right|_{\frac{\mu}{T}}=\left.\frac{\partial}{\partial U}\left[\frac{\partial}{\partial\left(\frac{P}{T}\right)}\right] \mathcal{H}\right|_{\frac{\mu}{T}} ;$

$\left.\frac{\partial}{\partial U} \mathcal{H}\right|_{\frac{\mu}{T}}=\frac{1}{T}$ e $\left.\frac{\partial}{\partial\left(\frac{P}{T}\right)} \mathcal{H}\right|_{\frac{\mu}{T}}=-V ;$

$$
\left.\frac{\partial}{\partial\left(\frac{P}{T}\right)}\left(\frac{1}{T}\right)\right|_{\frac{\mu}{T}}=-\left.\frac{\partial}{\partial U} V\right|_{\underline{T}} .
$$

\subsubsection{O Potencial Anônimo $\phi$}

$\phi=f\left(U, V, \frac{\mu}{T}\right)$.

(a) $\left.\frac{\partial}{\partial\left(\frac{\mu}{T}\right)}\left(\frac{\partial}{\partial V}\right) \phi\right|_{U}=\left.\frac{\partial}{\partial V}\left[\frac{\partial}{\partial\left(\frac{\mu}{T}\right)}\right] \phi\right|_{U}$;

$\left.\frac{\partial}{\partial V} \phi\right|_{U}=\frac{P}{T}$ e $\left.\frac{\partial}{\partial\left(\frac{\mu}{T}\right)} \phi\right|_{U}=N ;$ 


$$
\left.\frac{\partial}{\partial V} N\right|_{U}=\left.\frac{\partial}{\partial\left(\frac{\mu}{T}\right)}\left(\frac{P}{T}\right)\right|_{U}
$$

(b) $\left.\frac{\partial}{\partial\left(\frac{\mu}{T}\right)}\left(\frac{\partial}{\partial U}\right) \phi\right|_{V}=\left.\frac{\partial}{\partial U}\left[\frac{\partial}{\partial\left(\frac{\mu}{T}\right)}\right] \phi\right|_{V}$;

$\left.\frac{\partial \Phi}{\partial U}\right|_{V}=\frac{1}{T}$ e $\left.\frac{\partial \Phi}{\partial\left(\frac{\mu}{T}\right)}\right|_{V}=N$;

$$
\left.\frac{\partial}{\partial\left(\frac{\mu}{T}\right)}\left(\frac{1}{T}\right)\right|_{V}=\left.\frac{\partial}{\partial U} N\right|_{V}
$$

(c) $\left.\frac{\partial}{\partial V}\left(\frac{\partial}{\partial U}\right) \phi\right|_{\frac{\mu}{T}}=\left.\frac{\partial}{\partial U}\left(\frac{\partial}{\partial V}\right) \phi\right|_{\frac{\mu}{T}} ;$

$\left.\frac{\partial}{\partial U} \phi\right|_{\frac{\mu}{T}}=\frac{1}{T}$ e $\left.\frac{\partial}{\partial V} \phi\right|_{\frac{\mu}{T}}=\frac{P}{T} ;$

$$
\left.\frac{\partial}{\partial V}\left(\frac{1}{T}\right)\right|_{\frac{\mu}{T}}=\left.\frac{\partial}{\partial U}\left(\frac{P}{T}\right)\right|_{\frac{\mu}{T}} \cdot
$$

\subsubsection{O Potencial Anônimo ("Potencial de Boltzmann") $\mathcal{F}$}

$\mathcal{F}=f\left(V, \frac{1}{T}, \frac{\mu}{T}\right)$.

(a) $\left.\frac{\partial}{\partial V}\left[\frac{\partial}{\partial\left(\frac{1}{T}\right)}\right] \mathcal{F}\right|_{\frac{\mu}{T}}=\left.\frac{\partial}{\partial\left(\frac{1}{T}\right)}\left(\frac{\partial}{\partial V}\right) \mathcal{F}\right|_{\frac{\mu}{T}}$;

$\left.\frac{\partial}{\partial\left(\frac{1}{T}\right)} \mathcal{F}\right|_{\frac{\mu}{T}}=-U$ e $\left.\frac{\partial}{\partial V} \mathcal{F}\right|_{\frac{\mu}{T}}=\frac{P}{T} ;$

$$
\left.\frac{\partial}{\partial V} U\right|_{\frac{\mu}{T}}=-\left.\frac{\partial}{\partial\left(\frac{1}{T}\right)}\left(\frac{P}{T}\right)\right|_{\frac{\mu}{T}} .
$$

(b) $\left.\frac{\partial}{\partial V}\left[\frac{\partial}{\partial\left(\frac{\mu}{T}\right)}\right] \mathcal{F}\right|_{\frac{1}{T}}=\left.\frac{\partial}{\partial\left(\frac{\mu}{T}\right)}\left(\frac{\partial}{\partial V}\right) \mathcal{F}\right|_{\frac{1}{T}}$;

$\left.\frac{\partial}{\partial\left(\frac{\mu}{T}\right)} \mathcal{F}\right|_{\bar{T}}=N$ e $\left.\frac{\partial}{\partial V} \mathcal{F}\right|_{\bar{T}}=\frac{P}{T}$

$$
\left.\frac{\partial}{\partial V} N\right|_{\frac{1}{T}}=\left.\frac{\partial}{\partial\left(\frac{\mu}{T}\right)}\left(\frac{P}{T}\right)\right|_{\frac{1}{T}} .
$$

(c) $\left.\frac{\partial}{\partial\left(\frac{1}{T}\right)}\left[\frac{\partial}{\partial\left(\frac{\mu}{T}\right)}\right] \mathcal{F}\right|_{V}=\left.\frac{\partial}{\partial\left(\frac{\mu}{T}\right)}\left[\frac{\partial}{\partial\left(\frac{1}{T}\right)}\right] \mathcal{F}\right|_{V}$;

$\left.\frac{\partial \mathcal{F}}{\partial\left(\frac{\mu}{T}\right)}\right|_{V}=N$ e $\left.\frac{\partial \mathcal{F}}{\partial\left(\frac{1}{T}\right)}\right|_{V}=-U$;

$$
\left.\frac{\partial}{\partial\left(\frac{\mu}{T}\right)} U\right|_{V}=-\left.\frac{\partial}{\partial\left(\frac{1}{T}\right)} N\right|_{V} .
$$




\subsubsection{O Potencial Nulo 0}

$0=f\left(\frac{1}{T}, \frac{\mu}{T}, \frac{P}{T}\right)$.

(a) $\left.\frac{\partial}{\partial\left(\frac{1}{T}\right)}\left[\frac{\partial}{\partial\left(\frac{P}{T}\right)}\right] 0\right|_{\frac{\mu}{T}}=\left.\frac{\partial}{\partial\left(\frac{P}{T}\right)}\left[\frac{\partial}{\partial\left(\frac{1}{T}\right)}\right] 0\right|_{\frac{\mu}{T}}$;

$\left.\frac{\partial}{\partial\left(\frac{P}{T}\right)} 0\right|_{\frac{\mu}{T}}=-V$ e $\left.\frac{\partial}{\partial\left(\frac{1}{T}\right)} 0\right|_{\frac{\mu}{T}}=-U$

$\left.\frac{\partial}{\partial\left(\frac{1}{T}\right)} V\right|_{\underline{\underline{\mu}}}=\left.\frac{\partial}{\partial\left(\frac{P}{T}\right)} U\right|_{\underline{\underline{\mu}}}$.

(b) $\left.\frac{\partial}{\partial\left(\frac{1}{T}\right)}\left[\frac{\partial}{\partial\left(\frac{\mu}{T}\right)}\right] 0\right|_{\frac{P}{T}}=\left.\frac{\partial}{\partial\left(\frac{\mu}{T}\right)}\left[\frac{\partial}{\partial\left(\frac{1}{T}\right)}\right] 0\right|_{\frac{P}{T}}$;

$\left.\frac{\partial}{\partial\left(\frac{\mu}{T}\right)} 0\right|_{\frac{P}{T}}=N$ e $\left.\frac{\partial}{\partial\left(\frac{1}{T}\right)} 0\right|_{\frac{P}{T}}=-U ;$

$$
\left.\frac{\partial}{\partial\left(\frac{\mu}{T}\right)} U\right|_{\bar{T}}=-\left.\frac{\partial}{\partial\left(\frac{1}{T}\right)} N\right|_{\bar{T}}
$$

(c) $\left.\frac{\partial}{\partial\left(\frac{P}{T}\right)}\left[\frac{\partial}{\partial\left(\frac{\mu}{T}\right)}\right] 0\right|_{\frac{1}{T}}=\left.\frac{\partial}{\partial\left(\frac{\mu}{T}\right)}\left[\frac{\partial}{\partial\left(\frac{P}{T}\right)}\right] 0\right|_{\frac{1}{T}}$;

$\left.\frac{\partial}{\partial\left(\frac{\mu}{T}\right)} 0\right|_{\frac{1}{T}}=N$ e $\left.\frac{\partial}{\partial\left(\frac{P}{T}\right)} 0\right|_{\frac{1}{T}}=-V$;

$$
\left.\frac{\partial}{\partial\left(\frac{\mu}{T}\right)} V\right|_{\frac{1}{T}}=-\left.\frac{\partial}{\partial\left(\frac{P}{T}\right)} N\right|_{\frac{1}{T}}
$$

\subsection{Otimização das Equações de Maxwell para as Funções Características de Massieu}

As vinte e quatro expressões da Seção 5.1 (Equação 68 a 91) pertenceriam ao conjunto das Identidades de Maxwell da Termodinâmica na representação da Entropia. Mas, para cada uma dessas expressões há outra expressão que representa o mesmo resultado, apesar das suas variáveis mantidas constantes serem distintas. Logo, a rigor, seriam necessárias apenas doze expressões. Mas, ao reagrupar as doze equações restantes, pode ser observado que ainda há repetição para cada uma dessas doze equações restantes, porém com derivadas parciais invertidas. Com isso, mais seis equações podem ser desprezadas, de modo a restarem apenas seis equações realmente distintas. Finalmente, seguem as Identidades de Maxwell da Termodinâmica que restaram após a eliminação das equações equivalentes: 


$$
\begin{aligned}
& \left.\frac{\partial}{\partial U}\left(\frac{P}{T}\right)\right|_{N, \frac{\mu}{T}}=\left.\frac{\partial}{\partial V}\left(\frac{1}{T}\right)\right|_{N, \frac{\mu}{T}} ; \\
& \left.\frac{\partial}{\partial U}\left(\frac{\mu}{T}\right)\right|_{V, \frac{P}{T}}=-\left.\frac{\partial}{\partial N}\left(\frac{1}{T}\right)\right|_{V, \frac{P}{T}} ; \\
& \left.\frac{\partial}{\partial V}\left(\frac{\mu}{T}\right)\right|_{U, \frac{1}{T}}=-\left.\frac{\partial}{\partial N}\left(\frac{P}{T}\right)\right|_{U, \frac{1}{T}} ; \\
& \left.\frac{\partial}{\partial\left(\frac{1}{T}\right)}\left(\frac{\mu}{T}\right)\right|_{V, \frac{P}{T}}=\left.\frac{\partial}{\partial N} U\right|_{V, \frac{P}{T}} ; \\
& \left.\frac{\partial}{\partial N} V\right|_{\frac{1}{T}, U}=\left.\frac{\partial}{\partial\left(\frac{P}{T}\right)}\left(\frac{\mu}{T}\right)\right|_{\frac{1}{T}, U} ; \\
& \left.\frac{\partial}{\partial U} V\right|_{N, \frac{\mu}{T}}=-\left.\frac{\partial}{\partial\left(\frac{P}{T}\right)}\left(\frac{1}{T}\right)\right|_{N, \frac{\mu}{T}} .
\end{aligned}
$$

\section{DISCUSSÕES}

A Equação (44), denominada por "Relação de Euler da Termodinâmica" na representação da Entropia, é o resultado da razão do saldo da Energia Interna, após a retirada das energias Mecânica e Química, dividido pela Temperatura Absoluta. Ou ainda, a Entropia de um Sistema é o resultado a razão da Energia Térmica pela Temperatura Absoluta. Com isso, ao retirar as energias Mecânica e Química de um Sistema Simples, restará nesse sistema a Energia Térmica. Pelo fato da Energia Térmica ser degradável no decorrer da sua transformação, não estará mais integramente disponível para ser transferida entre Sistemas para realização de Trabalho. É nesse sentido que a Entropia está associada à degradação da energia de um Sistema.

Observe que no Cubo da Figura 1 que as Setas Arestas divergem da Entropia $S$ e convergem para o Potencial Nulo 0. Ao deslocar de um potencial a outro pelas arestas do cubo, sempre num mesmo sentido das Setas Arestas, o Sistema Termodinâmico atingirá um estado de potencial sempre menor que o potencial anterior. Após a retirada de cada parcela de energia, dividida pela sua Temperatura Absoluta, o sistema atingirá o último estado de potencial nulo, denominado por Potencial Sumidouro, dando o significado físico ao jargão "reduzir um sistema a nada" (SÁ MARTINS, 2014).

Revista RBBA Revista Binacional Brasil Argentina Vol. 10, num. 2, dez/2021, p. 365-389 
CARACTERÍSTICAS DE MASSIEU

\section{CONCLUSÕES}

As grandezas potenciais da Termodinâmica, na representação da Energia, são também denominadas por "Potenciais Termodinâmicos". Das oito grandezas que têm essa denominação, quatro delas são dos sistemas fechados, enquanto as outras quatro são dos sistemas abertos. Esses “potenciais” para os sistemas fechados são a Energia Interna, a Entalpia, a Energia Livre de Gibbs e a Energia Livre de Helmholtz. Já as grandezas dos sistemas abertos são o Potencial de Landau, o Potencial Nulo e dois Potenciais Anônimos (CORREIA, 2021b). Nesse sentido, o uso do termo "potencial Termodinâmico" não é garantia que se trata de um potencial termodinâmico, de forma que os Potenciais Termodinâmicos propriamente ditos são os que estão descritos na representação da Entropia.

Finalmente, o diagram cúbico na representação da Entropia apresentado neste artigo visa facilitar a obtenção das equações dos Potenciais Termodinâmicos, de modo análogo ao modelo na representação da Energia proposto por Correia (2021b).

Embora exista um algebrismo matemático consolidado que equaciona as Leis e as Relações da Termodinâmica do Equilíbrio para Sistemas Simples, a partir do modelo ora proposto podem ser obtidas as mesmas equações e relações, porém sem utilizar, de forma explícita, as Transformadas de Legendre.

Neste sentido, o modelo S-Digraph aqui proposto constitui numa ferramenta educacional auxiliar que poderá contribuir ao ensino e à aprendizagem da Termodinâmica do Equilíbrio e à obtenção das interrelacões entre os Potenciais Termodinâmicos para sistemas abertos, minimizar esforços para equacionar as relações da Termodinâmica do equilíbrio, bem como dar significado aos Grandes Potenciais, uma vez que a literatura científica ainda os tratam com muita superficialidade.

Com base no que foi observado durante a elaboração deste artigo, a Termodinâmica para Sistemas Abertos ainda é um campo que necessita de investigação e análise epistemológica, carecendo de uma elaboração e de uma explanação teórica e fenomenológica mais concisa quanto à definição dos Grandes Potenciais da Termodinâmica. Apesar dos esforços e das contribuições de muitos pesquisadores, a abordagem dos Potenciais Termodinâmicos de Massieu ainda é pouco explorada pelos livros didáticos de Termodinâmica.

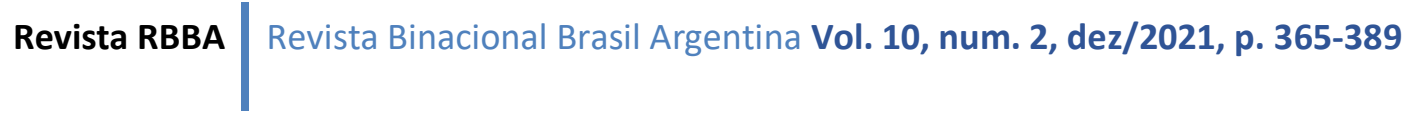




\section{REFERÊNCIAS}

ARENS, R. An Axiomatic Basis for Classical Thermodynamics. Journal of Mathematical Analysis and Applications. 6, 207-229. 1963. Disponível em https://www.sciencedirect.com/science/article/pii/0022247X63900052. Acesso em 02 jan. 2020.

BALIAN, R. François Massieu et les Potentiels Thermodynamiques. Histoire des Sciences/ Évolution des Disciplines et Histoire des Découvertes. 2015, 6 pp. Disponível em https://www.academie-sciences.fr/pdf/hse/evol_Balian2.pdf. Acesso em 13 fev. 2021.

BALIAN, R. François Massieu and the Thermodynamic Potential. Comptes Rendus Physique. 18, 526-539. 2017.

BRAGA, J. P. A Formulação ab-initio da Segunda Lei da Termodinâmica. Química Nova, 21(4), 1998. Disponível em http://www.scielo.br/pdf/qn/v21n4/3200.pdf. Acesso em 02 jan. 2020 .

CALLEN, H. B. Thermodynamics and a Introduction to Thermoestatistics, Second Edition, John Wiley and Sons, New York, 1985.

CORREIA, J.J.; MAGALHÃES, L. D. R.; LIMA, L. S. Obstáculos Epistemológicos e o Conceito de Calor. Scientibus Série Ciências Físicas 04: 1-10, 2008. Disponível em: http://periodicos.uefs.br/index.php/SSCF/article/view/SSCF-v.4-A1. Acesso em: 30 jun. 2021. DOI: http://dx.doi.org/10.13102/sscf.v4i0.5270

CORREIA, J. J.; OLIVEIRA, W. C. A Definição de Entalpia em Livros Didáticos. Revista Binacional Brasil-Argentina. v. 8, n. 1, p. 327-363, mai. 2019. DOI: https://doi.org/10.22481/rbba.v08i1.4912.

CORREIA, J. J.; GUIMARÃES, G. F. Definição da Energia Livre de Helmholtz em Fontes Didáticas. Revista Binacional Brasil-Argentina. v. 8, n. 2, p. 127-155, mar. 2020a. DOI: https://doi.org/10.22481/rbba.v08i2.7869.

CORREIA, J. J.; GUIMARÃES, G. F. Definição da Energia Livre de Gibbs em Fontes Didáticas. Caderno de Física da UEFS. v. 18, n. 2, p. 1-25, 2020 b.

CORREIA, J. J.; GUIMARÃES, G. F. Definição de Potencial Químico em Fontes Didáticas. Revista Binacional Brasil-Argentina. v. 10, n. 12, p. 412-438, jun. 2021a. DOI: https://doi.org/10.22481/rbba.v10i01.8003.

CORREIA, J. J. An E-DIGRAPH for the Thermodynamic Potential Energies. No prelo. 2021b.

DEBENEDETTI, P. G. Generalized Massieu-Planck Functions: Geometric Representation, Extrema and Uniqueness Porperties. Journal of Chemical Physics. 85 (4) 2132, 1986. DOI: https://doi.org/10.1063/1.451105.

FOX, R. F. J. Chem. Educ. 53, 7. 1976.

GIBBS, J. W. Collected Works, Vol. I, Thermodynamics, Longman, New York, 1928.

KERR, W. C.; MACOSKO, J. C. Thermodynamic Venn Diagrams: Sorting out forces, fluxes, and Legendre transforms. American Journal of Physics. 79 (9), 950. 2011. Disponível em https://www.researchgate.net/publication/235736528_Thermodynamic_Venn_diagrams_Sorti 
ng_out_forces_fluxes_and_Legendre_transforms. Acesso em 18 de abril de 2020. DOI: https://doi.org/10.1119/1.3599177.

KOCIK, J.: On Geometry of Pheonomenological Thermodinamics. Jul. 2018. Disponível em: https://www.researchgate.net/publication/326586312_On_geometry_of_phenomenological_th ermodynamics. Acesso em 25 dez. 2019.

KOENIG, F. O. Families of Thermodynamic Equations. I The Method of Transformations by the Characteristic Group. Journal of Chemical Physics. 3, 29. 1935.

KOENIG, F. O. Families of Thermodynamic Equations. II. The Case of Eight Characteristic Functions. Journal of Chemical Physics. 56, 4556. 1972.

LOBO, L. Q.; FERREIRA, A. G. M. Termodinâmica e Propriedades Termofísicas. Imprensa da Universidade de Coimbra, 2006. Disponível em http://dx.doi.org/10.14195/978-989-260487-9. Acesso em 11 jul. 2021.

PÁDUA, A. B.; PÁDUA, C; G.; SILVA, J. L. C.; MARTINS, R. S.; POSTALI, F. B.; TIRITAN, L. A. C. Termodinâmica Clássica ou Termodinâmica do Equilíbrio: aspectos conceituais básicos. Semina: Ciências Exatas e Tecnológicas. v. 29, n. 1, p. 57-84. 2008.

PATE, S. F. The thermodynamic cube: A mnemonic and learning device for students of classical thermodynamics. American Journal of Physics. 67, 1111. 1999.

POGLIANI, L. Digrasphs, Graphs and Thermodynamics Equations. In: HAGHI, A. K. et al (org.) Applied Chemistry and Chemical Engineering, Vol. I - Mathematical and Analytical Techniques. Apple Academic Press, Canadá, 2018.

SÁ MARTINS, J. S de. Aula 2.5 - [Termodinâmica] Processos a pressão constante, calor latente e entalpia. Instituto de Física da Universidade Federal Fluminense (UFF). Rio de Janeiro. Youtube, videoaulas UFF, 2014. Disponível em: https://www.youtube.com/watch?v=2CkJ1iJ3nBI\&list=PLJHqUYUf9YoopjoVLiuc1jj3YSiT 9yzpl\&index=5. Acesso em in: 25 jan 2019.

SOUZA FILHO, O. M. Cadernos de História e Filosofia da Ciência, Campinas, Série 3, v. 8, n. 1, p. 79-140, jan.-jun. 1998.

ZHAO, J. -C. A Minemonic Scheme for Thermodynamic. Materials Reseach Society Bulletin. V. 34, fevereiro de 2009. Disponível em http://web.mit.edu/course/8/8 .593/spring 10/bk/on_MRS_Thermodynamic_Mnemonic.pdf. Acesso em 16 abr. 2020. 